\title{
Probable Values of Current Solar Cycle Peak
}

\author{
V. M. Silbergleit ${ }^{1,2}$ \\ ${ }^{1}$ Consejo Nacional de Investigaciones Científicas y Técnicas (CONICET), Buenos Aires, Argentina \\ ${ }^{2}$ Facultad de Ingeniería, Universidad de Buenos Aires, Av. Las Heras 2214-Piso 3-C1127AAR, Buenos Aires, Argentina
}

Correspondence should be addressed to V. M. Silbergleit, vms10ar@gmail.com

Received 18 November 2011; Revised 27 December 2011; Accepted 3 January 2012

Academic Editor: J. P. Rozelot

Copyright ( 2012 V. M. Silbergleit. This is an open access article distributed under the Creative Commons Attribution License, which permits unrestricted use, distribution, and reproduction in any medium, provided the original work is properly cited.

\begin{abstract}
An analysis of multiple linear regression method applied to solar cycles 4 to 23 using lagged values of smoothed monthly mean sunspot numbers as independent variables is presented. According to that, the amplitude of current solar cycle 24 is estimated providing a quantitative prediction result. Our adjustment shows that the current cycle would have a sunspot peak less than the biggest one observed during the cycle 19 giving an additional support to the declination in solar activity which is currently happening.
\end{abstract}

\section{Introduction}

Good predictions of the intenseness of solar activity are increasing by considering satellites information. Such satellites often provide determinative links in communications as well as defense and are also often origin of important scientific information. The enlargement of ultraviolet emission from the Sun at times of high solar activity heats the Earth's upper atmosphere, which in turn causes the spread and enlargement of the influence on these satellites. The predictions of solar activity are important for technology, including the goodness of the operations of low-Earth orbiting satellites, electric power transmission grids, and high-frequency radio communications, among other problems. Long-term predictions of solar activity are therefore extremely important to auxiliary plan missions and to project satellites that will remain active for their available lifetime.

As we know, the solar magnetism is the route to understanding the processes involved. The Sun's differential rotation, meridional circulation, and large-scale convective motions all contribute to produce the cyclic magnetic development observed. So far we have not produced theories that combine these mechanisms in a model, then we are necessitated to predict solar activity by statistical methods that depend on determining correlations between past and future behavior. Numerous studies to predict the maximum solar activity level have been published (e.g., [1-6]). The general trend observed in recent solar activity cycles is toward larger amplitude sunspot cycles. Some articles use the analysis of the time series of historical spot numbers to obtain a power spectrum of periodicities. On the other hand, there is evidence that shows some kind of regularity in sunspot cycle modulation. The sunspot numbers present periodicities of 11 years (Schwabe cycle), 22 years (Hale cycle), and 88 years (Gleissberg cycle). As it was published by [7] the duration of each cycle could be associated with a variation in the solar energy output.

Reading the above-mentioned articles, we found several interesting topics related to the three main groups of prediction methods.

(1) Precursor methods depend on the value of some data of solar activity or magnetism at a definite time to predict the amplitude of the following solar maximum. They concluded that each numbered solar cycle is a consistent unit in itself, while solar activity appears consist of a series of less compact intercorrelated particular cycles.

(2) Extrapolation methods are based on the previous history that the physical process giving rise to the sunspot number register is statistically homogeneous; that is, the mathematical regularities underlying its changes are the same at any point of time, and, 


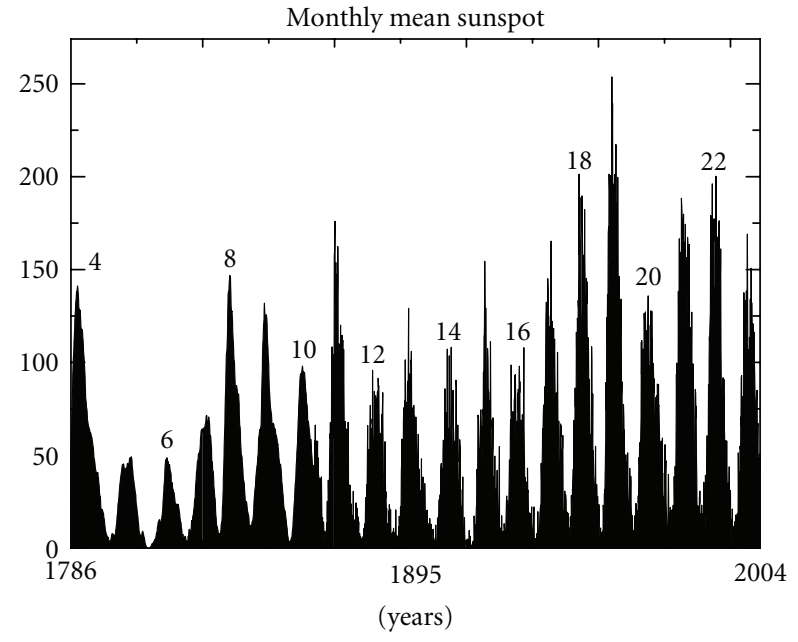

Figure 1: The sunspot cycle well documented over last 300 years shows a 11-year pattern of solar activity. Data from cycle 4 to cycle 23.

therefore, it lends itself to analysis and forecasting by time series methods.

(3) Finally, lieu of an analysis of measured data alone, model-based predictions use physically consistent dynamo models to predict solar activity.

During the last few solar cycles, precursor methods have clearly been superior to extrapolation methods. Thompson's method by the "precursor method" (see [3]) considers that the maximum amplitude of a cycle is proportional to the number of geomagnetic disturbed days in the cycle preceding it. It takes into consideration a solar dynamo theory hypotesis, where the polar field in the declining phase and at minimum is the origin of future toroidal field within the Sun that will cause solar activity. This method that has produced predictions according to the right range during the past few solar cycles was published by [8], whose approach is based on the polar field precursor. This method produces the solar physical union between the polar field, coronal holes, the interplanetary magnetic field, and the geomagnetic activity. The "precursor method" appears to be the best, but this is largely due to its accuracy in predicting the amplitude of cycle 19.

The beginning cycle 24 will probably mark the end of the Sun switching to a state of less strong activity. It will be an important proof for cycle prediction methods and for the understanding of the solar dynamo.

Physical processes contribute to producing the Sun's 11year activity cycle as the occurrence of coronal holes. Many models of the solar dynamo exist but none are complete. Part of the solution related to flows in the solar convection zone is studied by helioseismology and large-scale numerical models. The sun shows a roughly 11-year cycle of activity, from stormy to quiet and back again. Solar storms begin with complex magnetic fields generated by the sun's disturbing electrically charged gas. Solar magnetic fields can suddenly break, rejecting extremely great energy as a flare or a coronal mass ejection. This disturbed solar activity often occurs near sunspots.

Cycle 19 was the largest in recorded history (with 201.3 smoothed sunspot number at the maximum); cycles 21 and 22 both showed annual averages of geomagnetic activity that were large in comparison with most cycles in the record of an indices. Cycles 21 and 22 were the second and the third largest (with 164.5 and 158.5 smoothed sunspot number maximum, resp.). The important variability from one cycle to the next shows the difficulty in making empirical predictions of the Sun activity.

In this study, our goal is to describe a technique for predicting the amplitude of the solar cycles considering multiple regression method and using lagged values as independent variables. This technique will provide more reliable estimations of the level of solar activity several years into the future. One fundamental problem associated with solar cycle predictions is the small number of solar cycles that are well observed. Therefore most of our efforts at predicting solar cycle activity levels are obtained with the statistics of 10 or 20 cycles.

\section{Data, Regression Technique, and Results}

Over 300 years, the average number of sunspots has regularly increased and decreased in an approximately 11-year sunspot cycle. While some other aspects of the Sun vary distinctly over the years (e.g., coronal holes tend to be most numerous following sunspot maximum), the differential rotation of the Sun, southern circulation, and large-scale convective motions all play important roles in generating the cyclic magnetic behavior (see [9]).

For the present analysis we used monthly averages of the international sunspot number available from the National Geophysical Data Center (ftp://ftp.ngdc.noaa.gov/). Although these data extend from 1749 to the present, the nature of the data is: (a) poor for during 1700-1748, (b) doubtful during 1749-1817, (c) appropriate during 18181847 , and certain from 1848 to the present (see [10]). Values for years prior to 1849 are missing, thereby making that data less reliable. Taking into account the sunspot cycles plotted in Figures 1 and 2a, we can observe that the pairs: 4-5, 8-9, and 22-23 do not verify the "even-odd" effect. Thus, the analysis of the available solar data, including both statistical and physical indices, make it possible to assume that the integral even-odd effect and the rise rate-amplitude effect are the most prominent and universal statistical features of the solar activity cycles. The differences between odd and even solar cycles are a consequence of the nonlinear interactions that procure the stabilizing mechanism for the cycle's amplitude. If, for example, the magnetic field is larger than average for a given cycle (say odd), the nonlinear feedback machine can generate a magnetic field that is smaller than average for the next cycle (even), then one that is larger than average for the following cycle (odd), and so forth. As a consequence the odd cycles have larger amplitudes than even cycles in the sunspot records; it is less pronounced before 1823, which might be associated with lesser accuracy of the data [31]. 


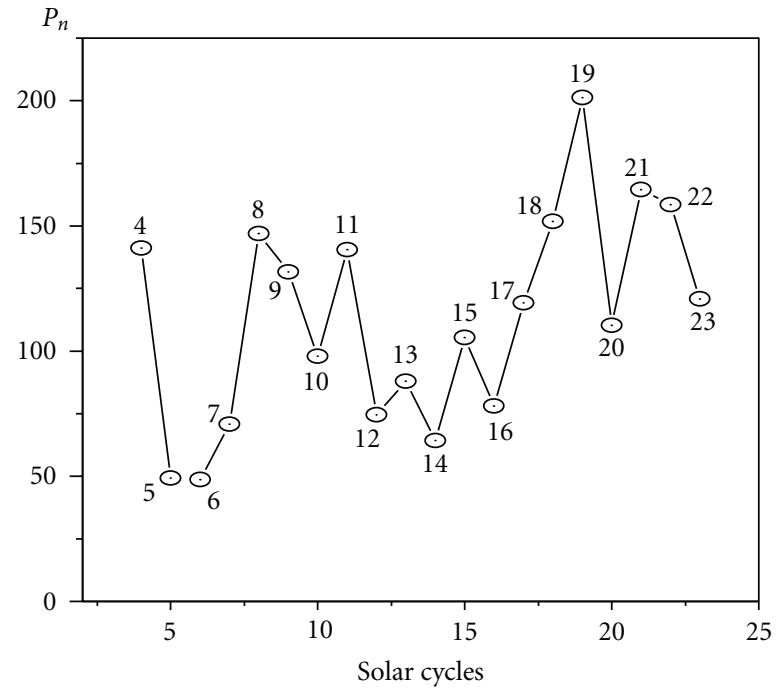

(a)

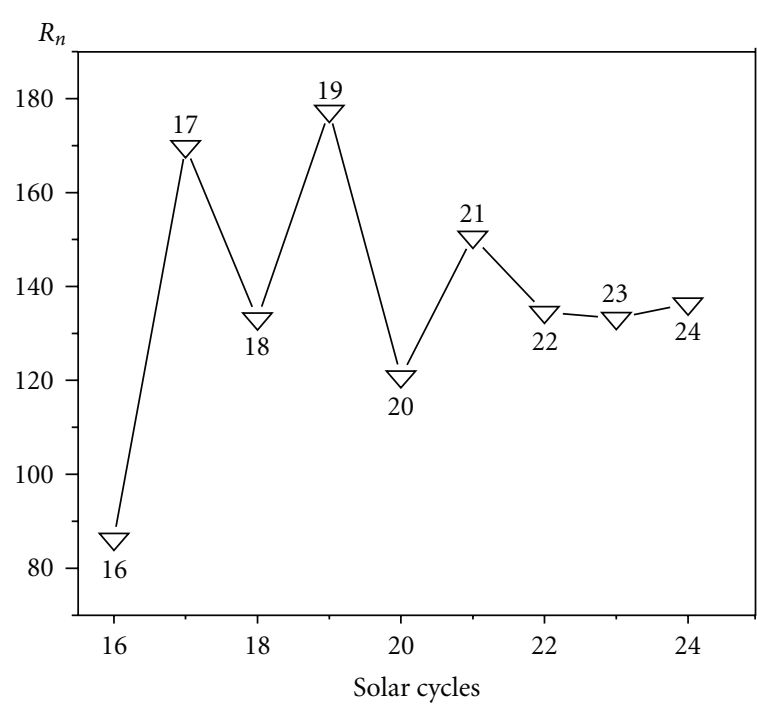

(b)

Figure 2: (a) Solar cycle amplitudes from cycle 4 to cycle 23 used to calculate the $R_{n}$. The filled circles are the observed sunspots $\left(P_{n}\right)$, and (b) Solar cycle peaks from cycles 16 to 24 as they are estimated. The filled triangles are the predicted sunspots $\left(R_{n}\right)$.

Sunspots appear on the Sun in two bands on either side of the equator that drift toward lower latitudes as each sunspot cycle progresses. The observation of the drift of the centroid of the sunspot area toward the equator in each hemisphere from 1874 to 2002 shows that the drift rate slows as the centroid approaches the equator. The comparison between the drift rate at sunspot cycle maximum and the period of each cycle for each hemisphere exhibits an extremely significant anticorrelation: hemispheres with faster drift rates have shorter periods. The drift rate at maximum is significantly correlated with the amplitude of the following cycle; a prediction of dynamo models that use a deep meridional flow toward the equator must be considered [32]. As cycles 22 and 23 were determined with good data quality, we inferred that processes into the Sun would be the most important reason for this behavior. Inspection of Figure 1 reveals that each cycle shows a broad range of temporal behavior, and the development of cycles 22-23 could be explained by considering that the Geissberg cycle (with a period of 88 years) is in the declining phase giving us an additional support to the declination in solar activity which is currently happening.

The sunspot cycle is a useful route to mark the changes in the Sun. Some cycles (like 5-6-7-10-12-14-16) are small in amplitude (about half the size of cycle 19), while others are considerably larger (like 18-19-21 and 22). Most cycles show asymmetries, with the rise to maximum being faster than the fall to minimum.

Reference [11] suggested a regression technique for predicting solar activity levels one year to the future. They used an average sunspot cycle made up of smoothed sunspot numbers starting with cycle 8 . The regression coefficients are obtained by minimizing the sum of RMS differences between predicted and observed variations from the mean
TABLe 1: Observed $\left(P_{n}\right)$ and predicted $\left(R_{n}\right)$ amplitudes.

\begin{tabular}{lccc}
\hline & $n$ & $P_{n}$ & $R_{n}$ \\
\hline$(1)$ & 16 & 78.1 & 86.2 \\
$(2)$ & 17 & 119.2 & 169.8 \\
$(3)$ & 18 & 151.8 & 133.1 \\
$(4)$ & 19 & 201.3 & 177.3 \\
$(5)$ & 20 & 110.3 & 120.8 \\
$(6)$ & 21 & 164.5 & 150.5 \\
$(7)$ & 22 & 158.5 & 134.5 \\
$(8)$ & 23 & 120.8 & 133.2 \\
$(9)$ & 24 & $104^{\mathrm{a}}$ & $136.3 \pm 30.0$ \\
\hline
\end{tabular}

${ }^{a}$ sunspot number observed on 11/12/30.

cycle. Several changes have been considered to make this method more accurate and useful (e.g., the method can be used recursively as it was adopted by the Space Environment Service Center in Boulder, Colorado); the MSAFE (Marshall Solar Activity Future Estimates) method described by [12] and used by [13].

A multiple linear regression (MLR) has the form:

$$
S_{k}=\alpha_{0}+\sum Y_{i} \alpha_{i}
$$

where the $\alpha_{i}$ are constants (with $i=1,2, \ldots, n$ and $i \neq k$ ) and the independent variables $Y_{i}$, are lagged values of $S_{k}$.

To select the delayed values to be used in (1) the autocorrelation analysis of each series was calculated, then the best combination was find. The parameters of the multiple regression equation were estimated by using the least squares method applied to the data observed from cycles 4-23. The final error was calculated as the sum of the square difference between the observed value and the predicted one. 
TABLE 2: Statistical results.

(a)

\begin{tabular}{lcccc}
\hline & \multicolumn{4}{c}{ Multiple Regression: independent data $P_{n-12}$ and $P_{n-7}$; dependent data $P_{n}$} \\
Parameter & Value & Error & $t$-value & Prob $>|t|$ \\
\hline$a_{0}$ & 283.89853 & 60.46631 & 4.69515 & 0.00536 \\
$a_{2}$ & -0.59136 & 0.28610 & -2.06696 & 0.09360 \\
$a_{1}$ & -0.86801 & 0.44384 & -1.95568 & 0.10788 \\
\hline
\end{tabular}

(b)

\begin{tabular}{lccc}
\hline$R$ & $R$-Square (COD) & Adj. $R$-Square Root- & MSE (SD) \\
\hline 0.74548 & 0.55574 & 0.37803 & 30.28774 \\
\hline
\end{tabular}

(c) ANOVA table.

\begin{tabular}{lccccc}
\hline Item & Degrees of freedom & Sum of squares & Mean square & $F$ statistic & Prob $>F$ \\
\hline Model & 2 & 5711.72512 & 2855.86256 & 3.09568 \\
Error & 5 & 4612.65363 & 922.53073 & & \\
Total & 7 & 10324.37875 & & \\
\hline
\end{tabular}

At the 0.005 level, the means are not significantly different.

Table 3: Predicted maximum amplitude (PMA) for solar cycle 24 as they were published.

\begin{tabular}{lccc}
\hline & Reference & Year & PMA values \\
\hline$(1)$ & {$[5]$} & 2003 & $(87.5 \pm 23.5)$ \\
$(2)$ & {$[10]$} & 2008 & $(94 \pm 44)$ \\
$(3)$ & {$[15]$} & 2001 & $(<50)$ \\
$(4)$ & {$[16]$} & 2002 & $(101.3 \pm 18.1)$ \\
$(5)$ & {$[17]$} & 2003 & $(100 \pm 30)$ \\
$(6)$ & {$[18]$} & 2003 & $(110 \pm 15)$ \\
$(7)$ & {$[19]$} & 2009 & $(145 \pm 7)$ \\
$(8)$ & {$[20]$} & 2011 & $(90 \pm 20)$ \\
$(9)$ & {$[21]$} & 2007 & $(142 \pm 24)$ \\
$(10)$ & {$[22]$} & 2008 & $(90 \pm 10)$ \\
$(11)$ & {$[23]$} & 2009 & $(78 \pm 10 \%)$ \\
$(12)$ & {$[24]$} & 2009 & $(87 \pm 5)$ \\
$(13)$ & {$[25]$} & 2011 & $(113.3)$ \\
$(14)$ & {$[26]$} & 2011 & $(65)$ \\
$(15)$ & {$[27]$} & 2010 & $(67 \pm 8)$ \\
$(16)$ & {$[28]$} & 2010 & $(131 \pm 20)$ \\
$(17)$ & {$[29]$} & 2008 & $(87 \pm 7)$ \\
$(18)$ & {$[30]$} & 2010 & $(84.5 \pm 23.9)$ \\
\hline
\end{tabular}

To predict the maximum activity of solar cycles (here called $R_{n}$ ), the MLR is applied to the observed solar peaks $\left(P_{n}\right) . R_{n}$ is related to the amplitudes of two prior cycles (lagged values, identified by $n_{1}$ and $n_{2}$ with values equal to 7 and 12, resp.). The expression to be considered is

$$
R_{n}=a_{0}+a_{1} P_{n-n_{1}}+a_{2} P_{n-n_{2}} .
$$

The constant values $a_{0}, a_{1}$, and $a_{2}$ are obtained by applying the MLR method to the data series from cycles 4 to 23. We considered $P_{n-n_{1}}$ and $P_{n-n_{2}}$ as independent variables and $R_{n}$ as the dependent variable.
The constant resulted to be: $a_{0}=284( \pm 60), a_{1}=$ $-0,87( \pm 0,44)$ and $a_{2}=-0,59( \pm 0,28)$. The correlation coefficient obtained was $R=0.75$ and a negligible probability that it is due to chance. Finally, (2) can be rewritten with the least error of prediction, as

$$
R_{n,}=284-0.87 P_{n-7},-0.59 P_{n-12} .
$$

The accuracy of the prediction based on (3) can be verified by looking for the results shown in Table 1 . The statistical test and partial and final results are shown in Table 2.

Figures 2(a) and 2(b) show the $P_{n}$ values for cycles 4 to 23 and the estimated $R_{n}$ values for cycles 16 to 24 , respectively. The plotted $R_{n}$ values have a mean sequence of upward (cycles 16 to 19) and downward (cycles 19 to 24) trends.

The present technique is appropriate to solve the problem of maximum amplitude prediction for solar cycles, and we have estimated each of the last 8 cycles with typical differences between calculated and observed data less than 24 in terms of sunspot number (for all the cycles except cycle 17 which presents a difference of 50.6). These differences are in the same order as the ones obtained by [3] in which the author used the "precursor" method.

\section{Discussion and Conclusions}

Multiple linear regression method is used to estimate cycles 16 to 24 amplitude maximum. From an analysis of solar cycle peaks for cycles 4 to 23 , a shorter recurrence trend of 7 or 12 cycles is observed. One is related to the Gleissberg period, and the other agrees with one of the periodicities in the $\mathrm{C}^{14}$ time record, which is associated with solar activity variation because changes in the $\mathrm{C}^{14}$ activity result primarily from varying degree of modulation of the galactic cosmic ray flux by the sun. Historical data show that during periods of depressed solar activity the atmospheric $\mathrm{C}^{14}$ was rising and 
at times when large sunspots were reported, $\mathrm{C}^{14}$ showed a tendency to decrease (see [14]). The estimated amplitudes of future solar cycles (as they are shown in Table 1) confirm that the present cycle would have a sunspot peak less than the biggest one observed during the cycle 19.

For maximum amplitude of solar cycle 24, some published articles show differences in the results as they are shown in Table 3.

Our result agrees with [1] which indicates a decline in solar activity since the recorded cycle 22 maximum. Also the "even-odd" effect was not verified for the pair, 22-23 giving an additional support to the declination in solar activity which is currently happening.

The method considered for the prediction of the maximum amplitude of solar cycles enables us to work out a reliable estimation for the size of the following cycle 24 as $136.3 \pm 30.0$ smoothed monthly mean sunspot numbers, some months in advance. Our study confirms that maximum amplitude of current solar cycle would be lower than the first largest one.

\section{Acknowledgments}

The author is grateful to unknown referees for helpful comments and suggestions. This work was partially supported by 20020100100227 (UBACYT) of Facultad de Ingeniería, Universidad de Buenos Aires and 11420090100258 of CONICET of Argentina.

\section{References}

[1] W. Gleissberg, "Probability laws of sunspot variations," Astrophysical Journal, vol. 96, p. 234, 1942.

[2] G. M. Brown and E. C. Butcher, "The use of abnormal quiet days in $\mathrm{Sq}(\mathrm{H})$ for predicting the magnitude of sunspot maximum at the time of preceding sunspot minimum," Planetary and Space Science, vol. 29, no. 1, pp. 73-77, 1981.

[3] R. Thompson, "A technique for predicting the amplitude of the solar cycle," Solar Physics, vol. 148, no. 2, pp. 383-388, 1993.

[4] D. H. Hathaway, R. M. Wilson, and E. J. Reichmann, "A synthesis of solar cycle prediction techniques," Journal of Geophysical Research A, vol. 104, no. 10, pp. 22375-22388, 1999.

[5] S. Duhau, "An early prediction of maximum sunspot number in solar cycle 24," Solar Physics, vol. 213, no. 1, pp. 203-212, 2003.

[6] V. M. Silbergleit and P. A. Larocca, "Geomagnetic activity and solar cycles," Advances in Space Research, vol. 36, no. 12, pp. 2384-2387, 2005.

[7] E. Friis-Christensen and K. Lassen, "Length of the solar cycle: an indicator of solar activity closely associated with climate," Science, vol. 254, no. 5032, pp. 698-700, 1991.

[8] K. H. Schatten and W. D. Pesnell, "An early dynamo prediction: cycle 23 is approximately cycle 22," Geophysical Research Letters, vol. 20, no. 20, pp. 2275-2278, 1993.

[9] D. H. Hathaway, R. M. Wilson, and E. J. Reichmann, "A synthesis of solar cycle prediction techniques," Journal of Geophysical Research A, vol. 104, no. 10, pp. 375-388, 1999.
[10] R. P. Kane, "Prediction of solar cycle maximum using solar cycle lengths," Solar Physics, vol. 248, no. 1, pp. 203-209, 2008.

[11] A. G. McNish and J. V. Lincoln, "Prediction of sunspot numbers," Eos, Transactions, American Geophysical Union, vol. 30, p. 673, 1949.

[12] K. O. Neihuss, H. C. Euler Jr., W. W. Vaugan et al., "Statistical technique for intermediate and long-range estimation of 13month smoothed solar flux and geomagnetic index," NASA Technical Reports TM-4759, 81, 1996.

[13] R. L. Holland and W. W. Vaughan, "Lagrangian least-squares prediction of solar flux (F-10.7)," Journal of Geophysical Research, vol. 89, no. 1, pp. 11-16, 1984.

[14] M. Stuiver, "Variations in radiocarbon concentration and sunspot activity," Journal Geophysical Research, vol. 66, no. 1, pp. 273-276, 1961.

[15] O. G. Badalyan, V. N. Obridko, and J. Sykora, "Brightness of the coronal green line and prediction for activity cycles 23 and 24," Solar Physics, vol. 199, no. 2, pp. 421-435, 2001.

[16] J. L. Wang, J. C. Gong, S. Q. Liu, G. M. Le, and J. L. Sun, "The prediction of maximum amplitudes of solar cycles and maximum amplitude of solar cycle 24," Chinese Journal Astronomy Astropysics, vol. 2, no. 6, pp. 557-562, 2002.

[17] K. H. Schatten, "Solar activity and the solar cycle," Advances in Space Research, vol. 32, no. 4, pp. 451-460, 2003.

[18] F. De Meyer, "A transfer function model for the sunspot cycle," Solar Physics, vol. 217, no. 2, pp. 349-366, 2003.

[19] I. J. Crosson and P. M. Binder, "Chaos-based forecast of sunspot cycle 24," Journal of Geophysical Research A, vol. 114, no. 1, Article ID A01108, 2009.

[20] R. P. Kane, "Prediction of the size of coming solar cycle 24 based on solar parameters during sunspot minimum between cycles 23 and 24," Indian Journal of Radio \& Space Physics, vol. 40, pp. 72-75, 2011.

[21] R. P. Kane, "A preliminary estimate of the size of the coming solar cycle 24, based on Ohl's precursor method," Solar Physics, vol. 243, no. 2, pp. 205-217, 2007.

[22] W. D. Pesnell, "Predictions of solar cycle 24," Solar Physics, vol. 252, no. 1, pp. 209-220, 2008.

[23] I. N. Kitashvili and A. G. Kosivichev, "Prediction of solar activity cycles by assimilating sunspot data into a dynamo model. Solar and stellar variability: impact on earth and planets," in Proceedings of the International Astronomical Union, no. 264, p. 202, IAU Symposium, 2009.

[24] A. Kilcik, C. N. K. Anderson, J. P. Rozelot, H. Ye, G. Sugihara, and A. Ozguc, "Nonlinear prediction of solar cycle 24," The Astrophysical Journal, vol. 693, no. 2, p. 1173, 2009.

[25] N. R. Rigozo, M. P. Souza Echer, H. Evangelista, D. J. R. Nordemann, and E. Echer, "Prediction of sunspot number amplitude and solar cycle length for cycles 24 and 25," Journal of Atmospheric and Solar-Terrestrial Physics, vol. 73, no. 11-12, pp. 1294-1299, 2010.

[26] A. Ajabshirizadeh, N. M. Jouzdani, and S. Abbassi, "Neural network prediction of solar cycle 24," Research in Astronomy and Astrophysics, vol. 11, no. 4, pp. 491-496, 2011.

[27] M. I. Pishkalo, "Prediciton of amplitude of solar cycle 24. Based on polar magnetic field of the sun at cycle minimum," Sun and Geosphere, vol. 5, no. 2, pp. 47-51, 2010.

[28] R. S. Dabas and K. Sharma, "Prediction of solar cycle 24 using geomagnetic precursors: validation and update," Solar Physics, vol. 266, no. 2, pp. 391-403, 2010. 
[29] J. Javaraiah, "Predicting the amplitude of a solar cycle using the north-south asymmetry in the previous cycle: II. An improved prediction for solar cycle 24," Solar Physics, vol. 252, no. 2, pp. 419-439, 2008.

[30] A. Yoshida and H. Yamagishi, "Predicting amplitude of solar cycle 24 based on a new precursor method," Annales Geophysicae, vol. 28, no. 2, pp. 417-425, 2010.

[31] B. R. Durney, "On the differences between odd and even solar cycles," Solar Physics, vol. 196, no. 2, pp. 421-426, 2000.

[32] D. H. Hathaway and L. Rightmire, "Variations in the Sun's meridional flow over a solar cycle," Science, vol. 327, no. 5971, pp. 1350-1352, 2010. 

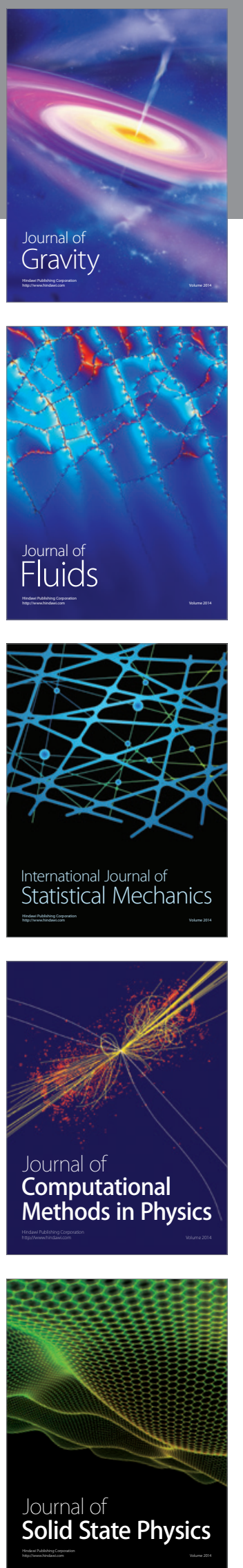

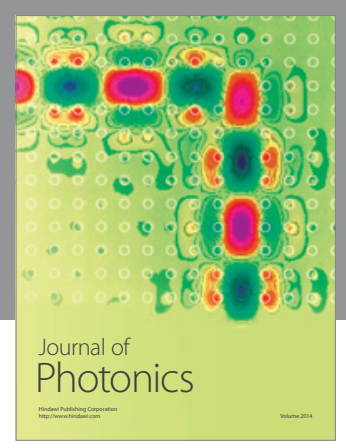

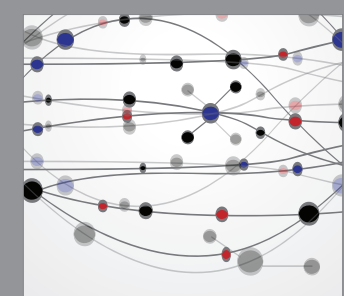

The Scientific World Journal
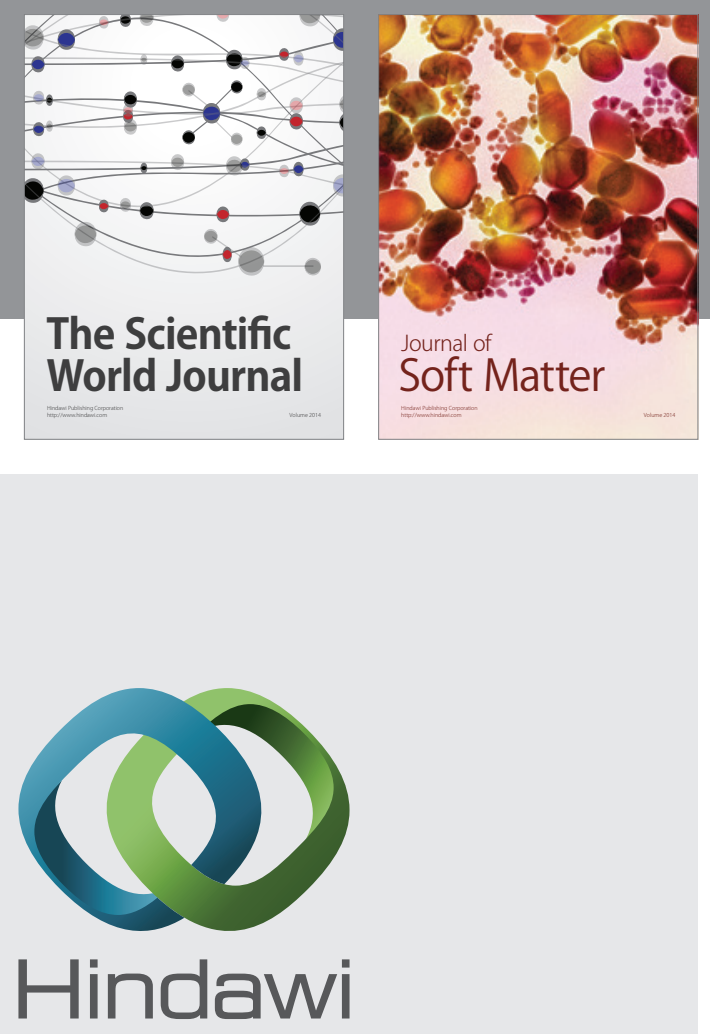

Submit your manuscripts at

http://www.hindawi.com
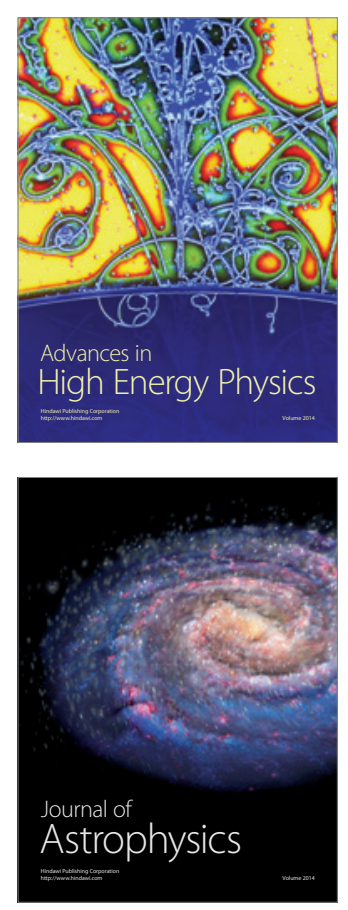
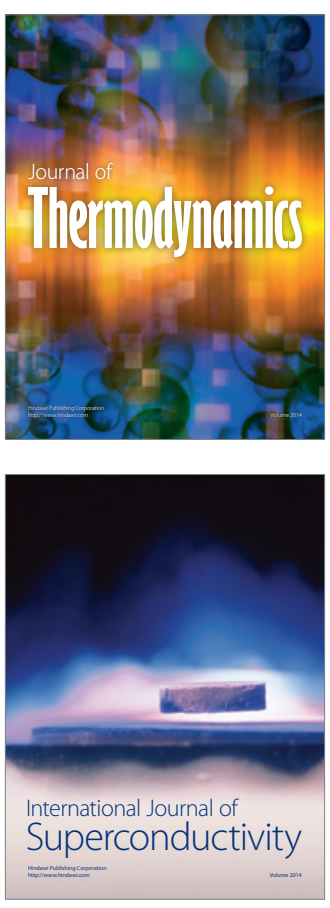
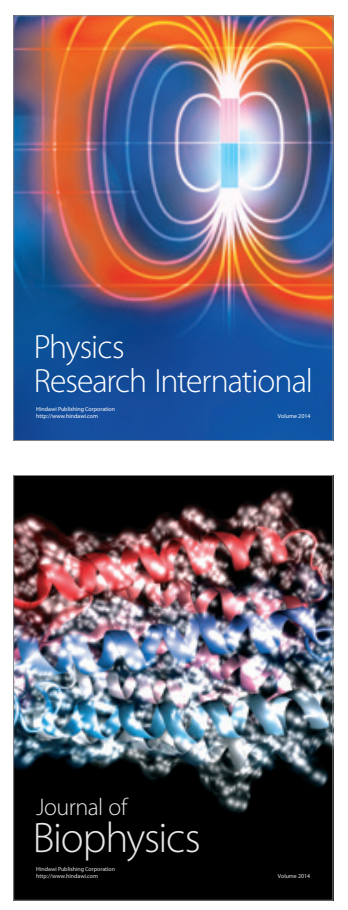
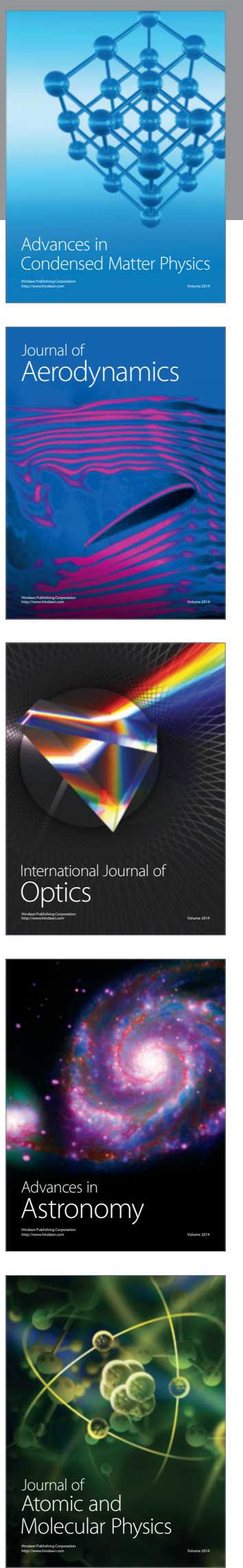